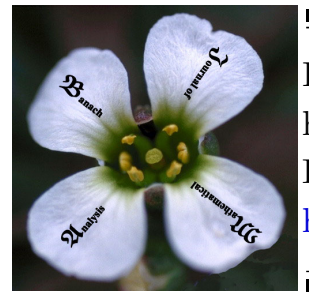

Banach J. Math. Anal. 9 (2015), no. 2, 196-200

http://doi.org/10.15352/bjma/09-2-14

ISSN: $1735-8787$ (electronic)

http://projecteuclid.org/bjma

\title{
A SHORT PROOF OF BURNSIDE'S FORMULA FOR THE GAMMA FUNCTION
}

\author{
CONSTANTIN P. NICULESCU ${ }^{1 *}$ AND FLORIN POPOVICI ${ }^{2}$ \\ Communicated by Zs. Pales
}

AbStRACt. We present simple proofs for Burnside's asymptotic formula and for its extension to positive real numbers.

\section{INTRODUCTION}

Burnside's asymptotic formula for factorial $n$ asserts that

$$
n ! \sim \sqrt{2 \pi}\left(\frac{n+1 / 2}{e}\right)^{n+1 / 2},
$$

in the sense that the ratio of the two sides tends to 1 as $n \rightarrow \infty$. This provides a more efficient estimation of the factorial, comparing to Stirling's formula,

$$
n ! \sim \sqrt{2 \pi} n^{n+1 / 2} e^{-n} .
$$

Indeed, for $n=100$, the exact value of 100 ! with 24 digits is

$$
\text { 9. } 332621544394415268169924 \times 10^{157} \text {. }
$$

Burnside's formula yields the approximation

$$
100 ! \approx 9.336491570312414838264959 \times 10^{157},
$$

while Stirling's formula is less precise, offering only the approximation

$$
100 ! \approx 9.324847625269343247764756 \times 10^{157} .
$$

Date: Received: Apr. 28, 2014; Accepted: Aug. 11, 2014.

* Corresponding author.

2010 Mathematics Subject Classification. Primary 33B15; Secondary 26B25, 41A30.

Key words and phrases. Burnside's asymptotic formula, Gamma function, log-convex function. 
The aim of the present paper is to present a short (and elementary) proof of Burnside's asymptotic formula and to extend it to positive real numbers. The main ingredients are Wallis' s product formula for $\pi$ and the property of logconvexity of the Gamma function.

\section{The PROOF OF Burnside's Formula}

The starting point is the following result concerning the monotonicity of the function $\left(1+\frac{1}{x}\right)^{x+\alpha}$ on the interval $[1, \infty)$.

Lemma 2.1. (I. Schur [6], Problem 168, page 38). Let $\alpha \in \mathbb{R}$. The sequence $a_{\alpha}(n)=\left(1+\frac{1}{n}\right)^{n+\alpha}$ is decreasing if $\alpha \in\left[\frac{1}{2}, \infty\right)$, and increasing for $n \geq N(\alpha)$ if $\alpha \in(-\infty, 1 / 2)$.

According to Lemma 1 above, for $\alpha \in(0,1 / 2)$ arbitrarily fixed, there is a positive integer $N(\alpha)$ such that

$$
\left(1+\frac{1}{k}\right)^{k+\alpha}<e<\left(1+\frac{1}{k}\right)^{k+1 / 2}
$$

for all $k \geq N(\alpha)$. As a consequence,

$$
\prod_{k=n}^{2 n}\left(\frac{k+1}{k}\right)^{k+\alpha}<e^{n+1}<\prod_{k=n}^{2 n}\left(\frac{k+1}{k}\right)^{k+1 / 2},
$$

for all $n \geq N(\alpha)$, equivalently,

$$
\frac{(2 n+1)^{2 n+\alpha}}{n^{n+\alpha}} \cdot \frac{1}{(n+1) \cdots(2 n)}<e^{n+1}<\frac{(2 n+1)^{2 n+1 / 2}}{n^{n+1 / 2}} \cdot \frac{1}{(n+1) \cdots(2 n)} .
$$

This can be restated as

$$
\begin{aligned}
\frac{2^{2 n+\alpha}\left(n+\frac{1}{2}\right)^{n+1 / 2}\left(1+\frac{1}{2 n}\right)^{n+\alpha}}{\sqrt{n+\frac{1}{2}}} & \cdot \frac{n !}{(2 n) !}<e^{n+1} \\
& <\frac{2^{2 n+1 / 2}\left(n+\frac{1}{2}\right)^{n+1 / 2}\left(1+\frac{1}{2 n}\right)^{n+1 / 2}}{\sqrt{n+\frac{1}{2}}} \cdot \frac{n !}{(2 n) !},
\end{aligned}
$$

whence

$$
\begin{aligned}
& \frac{1}{\sqrt{2 n+1}} \cdot \frac{(2 n) ! !}{(2 n-1) ! !} \cdot \frac{2^{\alpha+1 / 2}\left(1+\frac{1}{2 n}\right)^{n+\alpha}}{\sqrt{e}}<n !\left(\frac{e}{n+\frac{1}{2}}\right)^{n+1 / 2} \\
& <\frac{1}{\sqrt{2 n+1}} \cdot \frac{(2 n) ! !}{(2 n-1) ! !} \cdot \frac{2\left(1+\frac{1}{2 n}\right)^{n+1 / 2}}{\sqrt{e}}
\end{aligned}
$$

for all $n \geq N(\alpha)$. Here $n ! !=n \cdot(n-2) \cdots 4 \cdot 2$ if $n$ is even, and $n \cdot(n-2) \cdots 3 \cdot 1$ if $n$ is odd. 
Taking into account Wallis's formula,

$$
\lim _{n \rightarrow \infty} \frac{2 \cdot 2 \cdot 4 \cdot 4 \cdots(2 n) \cdot(2 n)}{1 \cdot 3 \cdot 3 \cdot 5 \cdot 5 \cdots(2 n-1) \cdot(2 n-1) \cdot(2 n+1)}=\frac{\pi}{2}
$$

that is,

$$
\lim _{n \rightarrow \infty} \frac{1}{\sqrt{2 n+1}} \cdot \frac{(2 n) ! !}{(2 n-1) ! !}=\sqrt{\frac{\pi}{2}},
$$

we arrive easily at Burnside's formula for factorial $n$ :

$$
n ! \sim \sqrt{2 \pi}\left(\frac{n+1 / 2}{e}\right)^{n+1 / 2} .
$$

\section{The extension of Burnside's formula for the Gamma function}

Our next goal is to derive from Burnside's formula the following asymptotic formula for the Gamma function:

Theorem 3.1. (R. J. Wilton [7]). $\Gamma(x+1) \sim \sqrt{2 \pi}\left(\frac{x+1 / 2}{e}\right)^{x+1 / 2}$ as $x \rightarrow \infty$.

The proof of the above theorem will be done by estimating the function

$$
f(x)=\Gamma(x+1)\left(\frac{e}{x+1 / 2}\right)^{x+1 / 2},
$$

for large values of $x$. We shall need the following double inequality:

Lemma 3.2. $\lfloor x\rfloor ! x^{\{x\}} \leq \Gamma(x+1) \leq\lfloor x\rfloor !(\lfloor x\rfloor+1)^{\{x\}}$ for all $x \geq 1$.

Here $\lfloor x\rfloor$ denotes the largest integer less than or equal to $x$ and $\{x\}=x-\lfloor x\rfloor$.

Proof. Our argument is based on the property of log-convexity of the Gamma function:

$$
\Gamma((1-\lambda) x+\lambda y) \leq \Gamma(x)^{1-\lambda} \Gamma(y)^{\lambda},
$$

for all $x, y>0$ and $\lambda \in[0,1]$. See [5], Theorem 2.2.4, pp. 69-70.

If $x$ is a positive number, then $\lfloor x\rfloor+1 \leq x+1<\lfloor x\rfloor+2$, which yields

$$
x+1=(1-\{x\})(\lfloor x\rfloor+1)+\{x\}(\lfloor x\rfloor+2) .
$$

Therefore,

$$
\begin{aligned}
\Gamma(x+1) & \leq \Gamma(\lfloor x\rfloor+1)^{1-\{x\}} \Gamma(\lfloor x\rfloor+2)^{\{x\}} \\
& =\lfloor x\rfloor !^{1-\{x\}}(\lfloor x\rfloor+1) !^{\{x\}} \\
& \leq\lfloor x\rfloor !(\lfloor x\rfloor+1)^{x-\lfloor x\rfloor} .
\end{aligned}
$$

In a similar way, taking into account that $\lfloor x\rfloor+1=\{x\} x+(1-\{x\})(x+1)$, we obtain

$$
\lfloor x\rfloor !=\Gamma(\lfloor x\rfloor+1) \leq \Gamma(x)^{\{x\}} \Gamma(x+1)^{1-\{x\}}=\frac{\Gamma(x+1)}{x^{x-\lfloor x\rfloor},}
$$

whence $\lfloor x\rfloor ! x^{x-\lfloor x\rfloor} \leq \Gamma(x+1)$. The proof is done. 
According to Lemma 2,

$$
\begin{aligned}
f(x) & \geq\lfloor x\rfloor ! x^{\{x\}} \cdot \frac{e^{x+1 / 2}}{(x+1 / 2)^{x+1 / 2}} \\
& =\Gamma(\lfloor x\rfloor+1) \cdot \frac{e^{\lfloor x\rfloor+1 / 2}}{(\lfloor x\rfloor+1 / 2)^{\lfloor x\rfloor+1 / 2}} \cdot \frac{e^{\{x\}}(\lfloor x\rfloor+1 / 2)^{\lfloor x\rfloor+1 / 2} x^{\{x\}}}{(x+1 / 2)^{x+1 / 2}} \\
& =f(\lfloor x\rfloor) \cdot\left(\frac{\lfloor x\rfloor+1 / 2}{x+1 / 2}\right)^{\lfloor x\rfloor+1 / 2} \cdot e^{\{x\}} \cdot\left(\frac{x}{x+1 / 2}\right)^{\{x\}} \\
& =f(\lfloor x\rfloor) \cdot\left(\frac{x}{x+1 / 2}\right)^{\{x\}} \cdot\left(\frac{e}{\left(1+\frac{\{x\}}{\lfloor x\rfloor+1 / 2}\right)^{\frac{\lfloor x\rfloor+1 / 2}{\lfloor x\}}}}\right)^{\{x\}} \\
& \geq f(\lfloor x\rfloor) \cdot\left(\frac{x}{x+1 / 2}\right)^{\{x\}}
\end{aligned}
$$

Similarly,

$$
\begin{aligned}
f(x) & =\Gamma(x+1)\left(\frac{e}{x+1 / 2}\right)^{x+1 / 2} \\
& \leq\lfloor x\rfloor !\left(\frac{e}{x+1 / 2}\right)^{x+1 / 2}(\lfloor x\rfloor+1)^{\{x\}} \\
& =f(\lfloor x\rfloor)\left(\frac{\lfloor x\rfloor+1 / 2}{x+1 / 2}\right)^{\lfloor x\rfloor+1 / 2}\left(\frac{\lfloor x\rfloor+1}{x+1 / 2}\right)^{\{x\}} e^{\{x\}} \\
& =f(\lfloor x\rfloor)\left(\frac{e}{\left(1+\frac{\{x\}}{\lfloor x\rfloor+1 / 2}\right)^{\frac{\lfloor x\rfloor+1 / 2}{\{x\}}}}\right)^{\{x\}}\left(\frac{\lfloor x\rfloor+1}{x+1 / 2}\right)^{\{x\}} .
\end{aligned}
$$

The formulas $(L W)$ and $(R W)$ show that

$$
\lim _{x \rightarrow \infty} f(x)=\lim _{n \rightarrow \infty} f(n),
$$

and this fact combined with Burnside's formula $(B)$ allows us to conclude that the limit of $f$ at infinity is $\sqrt{2 \pi}$, that is,

$$
\lim _{x \rightarrow \infty} \Gamma(x+1)\left(\frac{e}{x+1 / 2}\right)^{x+1 / 2}=\sqrt{2 \pi} .
$$

This ends the proof of Wilton's asymptotic formula.

It seems very likely that the above technique can be adapted to cover more accurate asymptotic formulas such as that of Gosper [4],

$$
n ! \sim \sqrt{2 \pi\left(n+\frac{1}{6}\right)}\left(\frac{n}{e}\right)^{n}
$$

and of its extension to real numbers. This is also supported by our joint paper with D. E. Dutkay [3]. 
Additional information concerning the approximation of the Gamma function may be found in the recent paper of G. D. Anderson, M. Vuorinen and X. Zhang [1].

\section{REFERENCES}

1. G.D. Anderson, M. Vuorinen and X. Zhang, Topics in Special Functions III. In vol. Analytic Number Theory, Approximation Theory and Special Functions (G.V. Milovanović and M.Th. Rassias eds.), pp. 297-345, Springer, 2014.

2. W. Burnside, A rapidly convergent series for $\log N$ !, Messenger Math. 46 (1917), 157-159.

3. D.E. Dutkay, C.P. Niculescu and F. Popovici, A note on Stirling's formula for the Gamma function, Journal of Prime Research in Mathematics 8 (2012), 1-4.

4. R.W. Gosper, Decision procedure for indefinite hypergeometric summation, Proc. Natl. Acad. Sci. USA 75 (1978) 40-42.

5. C.P. Niculescu and L.-E. Persson, Convex Functions and Their Applications. A Contemporary Approach, CMS Books in Mathematics, Vol. 23, Springer-Verlag, New York, 2006.

6. G. Pólya and G. Szegö, Problems and Theorems in Analysis I: Series. Integral Calculus. Theory of Functions. Reprint of the 1978 edition. Classics in Mathematics, Springer-Verlag, Berlin-Heidelberg-New York, 1998.

7. J.R. Wilton, A proof of Burnside's formula for $\log \Gamma(x+1)$ and certain allied properties of the Riemann s-function, Messenger Math. 52 (1922), 90-93.

${ }^{1}$ The Academy of Romanian Scientists, Splaiul Independentei No. 54, Bucharest, RO-050094 ROMANIA.

E-mail address: cpniculescu@gmail.com

${ }^{2}$ College Grigore Moisil, Brasov, Romania.

E-mail address: popovici.florin@yahoo.com 